\title{
Photoelectron spin-flipping and texture manipulation in a topological insulator
}

\author{
Chris Jozwiak' ${ }^{1}$, Cheol-Hwan Park ${ }^{2,3}$, Kenneth Gotlieb ${ }^{4}$, Choongyu Hwang ${ }^{5}$, Dung-Hai Lee, ${ }^{2,5}$, \\ Steven G. Louie ${ }^{2,5}$, Jonathan D. Denlinger ${ }^{1}$, Costel R. Rotundu ${ }^{5}$, Robert J. Birgeneau ${ }^{2,5,6}$, \\ Zahid Hussain ${ }^{1}$ and Alessandra Lanzara ${ }^{2,5 \star}$
}

\begin{abstract}
Recently discovered materials called three-dimensional topological insulators ${ }^{1-5}$ constitute examples of symmetryprotected topological states in the absence of applied magnetic fields and cryogenic temperatures. A hallmark characteristic of these non-magnetic bulk insulators is their protected metallic Dirac fermion-like surface states. Electrons in these surface states are spin polarized with their spins governed by their momentum, resulting in a helical spin texture in momentum space $^{6}$. Spin- and angle-resolved photoemission spectroscopy has been the only tool capable of directly observing this central feature with simultaneous energy, momentum and spin sensitivity ${ }^{6-12}$. By using an innovative photoelectron spectrometer $^{13}$ with a high-flux laser-based light source, we discovered a surprising property of these surface electrons. We found that the spin polarization of the resulting photoelectrons can be manipulated in three dimensions through selection of the light polarization. These effects are due to the spin-dependent interaction of the helical surface electrons with light, which originates from strong spin-orbit coupling. Our results illustrate unusual scenarios in which the spin polarization of photoelectrons is completely different from that of the originating initial states. The results also provide the basis for a source of highly spin-polarized electrons with tunable polarization direction.
\end{abstract}

The topological electronic band structure of a bulk topological insulator ensures the presence of gapless surface electronic states with Dirac-like dispersions similar to graphene. Unlike graphene, the topological surface states are spin polarized, with their spins locked perpendicular to their momentum, forming helical spinmomentum textures ${ }^{6}$ (Fig. 1a). The presence of such helical Dirac fermions forms an operational definition of a three-dimensional topological insulator, and much of the excitement surrounding topological insulators involves the predicted exotic phenomena and potential applications of these metallic states ${ }^{4,5}$. These include magnetoelectric effects ${ }^{14}$, exotic quasiparticles (in a proximityinduced superconducting state) called Majorana fermions that are their own antiparticles ${ }^{15}$, and applications ranging from spintronics to quantum computing ${ }^{16}$. Establishing methods that are sensitive to these states and their predicted behaviours have therefore generated much interest ${ }^{6,17-20}$.

Angle-resolved photoemission spectroscopy (ARPES) directly maps the dispersions and Fermi surfaces of such electronic states in energy-momentum space. Spin-resolved ARPES also measures the spin polarization of the corresponding photoelectrons. Following a common assumption that electron spin is conserved in the photoemission process, the technique has been used to identify the presence of the predicted helical spin textures of topological surface states $^{6-12}$. Utilizing a high-efficiency spin-resolved photoelectron spectrometer ${ }^{13}$ and a high-intensity laser light source that enabled rapid high-resolution data acquisition, we have found surprising new features of the photoelectron spin texture in the prototypical topological insulator, $\mathrm{Bi}_{2} \mathrm{Se}_{3}$ (ref. 21). In particular, the results demonstrate strong dependence of the photoelectron spin polarization on the photon polarization, enabling its full manipulation. This markedly illustrates that spin-conservation, commonly assumed for photoemission, is invalid in these materials ${ }^{22}$.

Figure 1a,b shows standard ARPES data collected from a $\mathrm{Bi}_{2} \mathrm{Se}_{3}$ single crystal. The sharp surface states form the ring-like Fermi surface piece in Fig. 1a with the cone-like dispersion in Fig. 1b. The sample is n-doped, such that the bottom of the bulk conduction band falls below the Fermi level, forming the chunk of spectral weight in the centre of the surface-state cone ${ }^{12,23}$.

Figure 1c,d shows spin-resolved energy distribution curves (EDCs), or plots of photoelectron intensity as a function of binding energy at a particular momentum, corresponding to the line-cut marked in Fig. 1b. The EDCs are resolved into distinct channels for spin-up and -down photoelectrons. Here, the spin quantization axis is the $y$ axis. The corresponding spin polarization, or $P_{y}$, curves are shown below, and are a measure of the relative difference between the number of spin-up and -down photoelectrons according to $P_{y}=\left(I_{\uparrow}-I_{\downarrow}\right) /\left(I_{\uparrow}+I_{\downarrow}\right)$. The data were acquired with linearly polarized light in two distinct photon polarization geometries, in which the electric field vector, $\hat{\epsilon}$, was in the $x-z$ plane $(p-$ polarization) and along the $y$ axis (s-polarization), respectively (Fig. 1e and insets in Fig. 1c,d).

In the case of $p$-polarized light (Fig. 1c), the intensity peak is primarily spin-up, and its $P_{y}$ is nearly +1 . Thus, photoelectrons from the surface state near $E_{\mathrm{F}}$ with momentum $\left(k_{x}, k_{y}\right)=\left(-k_{\mathrm{F}}, 0\right)$ were strongly polarized up along the positive $y$ axis as labelled by the blue arrow in the inset. This is consistent with previous spin-ARPES measurements ${ }^{6-12}$ and with the predicted helical spin texture (Fig. 1a) where the surface-state spins are tangential to the Fermi surface contour with clockwise helicity. Remarkably, when the light polarization is rotated by $\pi / 2$ to $s$-polarization (Fig. 1d),

\footnotetext{
${ }^{1}$ Advanced Light Source, Lawrence Berkeley National Laboratory, Berkeley, California 94720, USA, ${ }^{2}$ Department of Physics, University of California, Berkeley, California 94720, USA, ${ }^{3}$ Department of Physics and Astronomy and Center for Theoretical Physics, Seoul National University, Seoul 151-747, Korea, ${ }^{4}$ Graduate Group in Applied Science and Technology, University of California, Berkeley, California 94720, USA, ${ }^{5}$ Materials Sciences Division, Lawrence Berkeley National Laboratory, Berkeley, California 94720, USA, ${ }^{6}$ Department of Materials Science and Engineering, University of California, Berkeley, California 94720, USA. *e-mail: ALanzara@lbl.gov.
} 


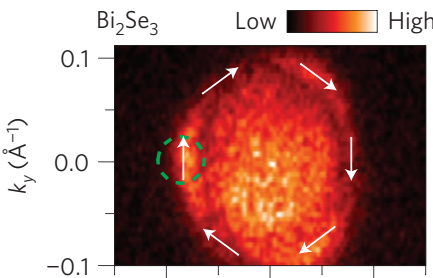

b

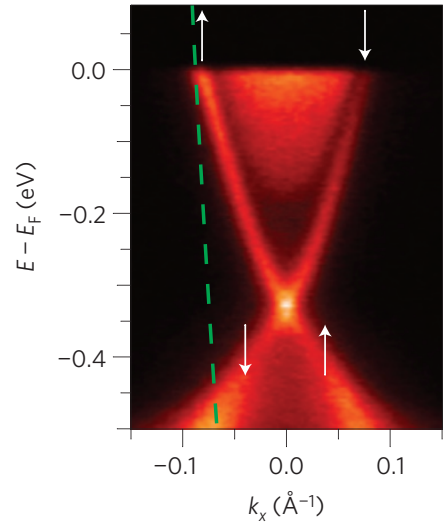

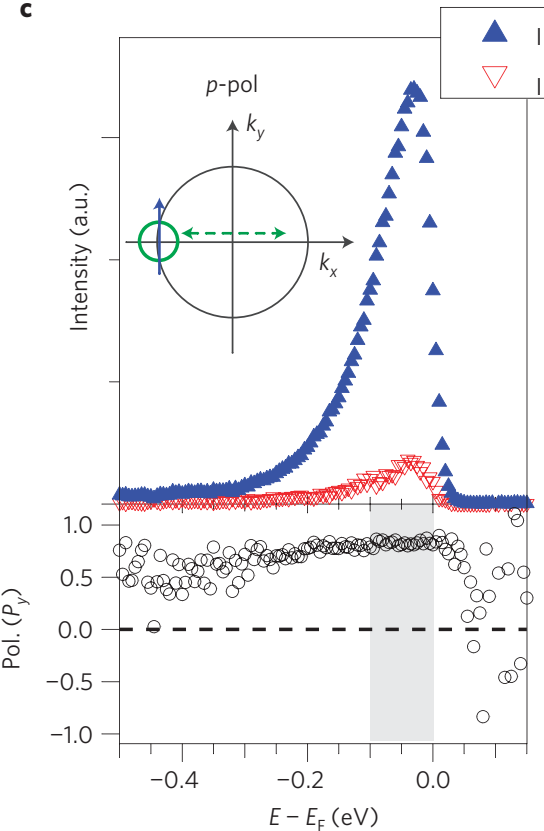

d

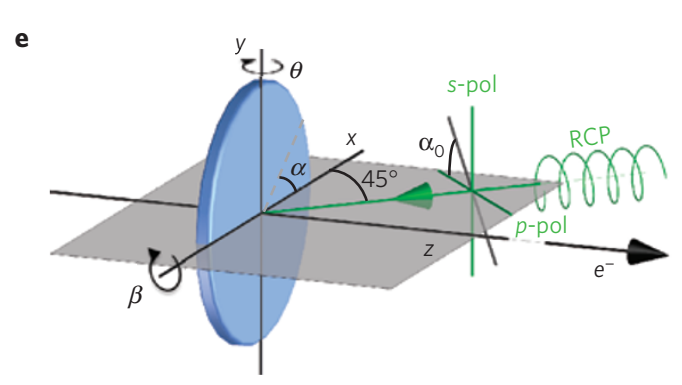

f

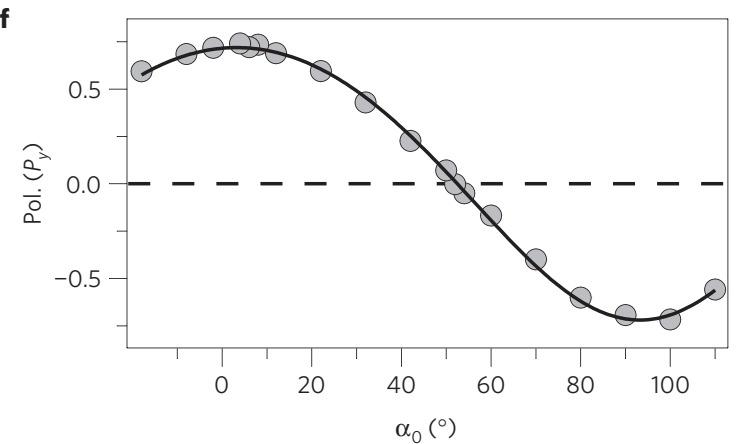

Figure 1 | The dependence of photoelectron spin on linear photon polarization observed in a topological insulator. $\mathbf{a}$, ARPES intensity map at $E_{\mathrm{F}}$ of the (111) surface of $\mathrm{Bi}_{2} \mathrm{Se}_{3}$, with the $\Gamma \mathrm{M}$ direction aligned along $k_{x}$. The white arrows show the expected spin polarization around the surface-state Fermi surface. b, ARPES intensity map as a function of binding energy and momentum. c, Spin-resolved photoemission intensity as a function of binding energy, at fixed emission angle ( $\sim$ fixed $\mathbf{k}$ ), corresponding to the dashed line-cut in $\mathbf{b}$, and the momentum location marked by the dashed circle in $\mathbf{a}$. The corresponding $y$ component of the photoelectron polarization, $P_{y}$, is shown in the bottom panel. The Fermi surface diagram inset highlights the $k$-space location, $\left(k_{x}, k_{y}\right)=\left(-k_{F}, 0\right)$ (green circle), along with the spin-polarization direction indicated by the data. The data are acquired with $p$-polarized photons, with the photon polarization vector, projected into the sample surface plane, shown as a dashed green arrow in the inset. $\mathbf{d}$, The same as in $\mathbf{c}$, but with s-polarized photons. e, Diagram of the experimental geometry. Linear polarization of photons can be continuously rotated as shown. Dashed grey line represents projection of incident light linear polarization on the sample surface. $\mathbf{f}$, Photoelectron spin polarization at $\left(k_{x}, k_{y}\right)=\left(-k_{F}, 0\right)$ as a function of rotation of the photon polarization. Photoelectron polarization is integrated in binding energy corresponding to the grey regions of the bottom panels in $\mathbf{c}, \mathbf{d}$. Black curve is a fit following the presented theory (see Supplementary Information).

the intensity peak reversed to primarily spin-down, with its $P_{y}$ nearly -1 , such that photoelectrons from the same initial state were polarized down as labelled by the red arrow in the inset (Fig. 1d). This is opposite to the expected spin texture for the surface states. Taken on its own, this result would counter previous spin-ARPES results, probably taken only with $p$-polarized light ${ }^{6,9,12}$, and seems to point to a spin texture of reversed helicity.

Furthermore, Fig. If shows the corresponding measured photoelectron polarization component, $P_{y}$, as a function of continuous rotation of the photon polarization vector between $p$ and s-polarizations. Clearly, the photoelectron spin polarization is dependent on the photon polarization and can be continuously modulated from nearly $P_{y}=+1$ to -1 .

The full energy and momentum dependence of this photoelectron spin flipping in $\mathrm{Bi}_{2} \mathrm{Se}_{3}$ is shown in Fig. 2 and is compared with the Rashba spin-split $\mathrm{Au}(111)$ Shockley surface state ${ }^{24}$. For reference, schematics of the theoretical spin-polarized surface band dispersion and Fermi surface spin texture of the $\mathrm{Bi}_{2} \mathrm{Se}_{3}$ and $\mathrm{Au}(111)$ surface states are shown in Fig. 2a,e, and the measured spin-integrated ARPES maps of the corresponding photoemitted electrons as a function of binding energy and $k_{x}$ are shown in Fig. 2b,f, respectively. Figure 2c,d (and Fig. 2g,h) shows the corresponding complete photoelectron $P_{y}$ maps for $p$ - and $s$-polarized light, respectively, for the $\mathrm{Bi}_{2} \mathrm{Se}_{3}$ (and $\mathrm{Au}$ ) surface state. In both cases, when the light is $p$-polarized, the photoelectron spin texture matches the expected surface-state spin texture (compare Fig. 2c with Fig. $2 \mathrm{a}$ and Fig. $2 \mathrm{~g}$ with Fig. $2 \mathrm{e}$ ). Specifically, for $\mathrm{Bi}_{2} \mathrm{Se}_{3}$, the photoelectrons following the branch of the Dirac cone with negative slope are spin-up (blue), and those along the branch with positive slope are spin-down (red), as expected. Fig. 2c is shown with an asymmetric colour scale to offset an overall shift in $P_{y}$ due to its particular experimental geometry (see Supplementary Information). Similarly, for the $\mathrm{Au}(111)$ surface state in Fig. 2g, the photoelectrons corresponding to the nearly free electron parabola shifted left are 
a

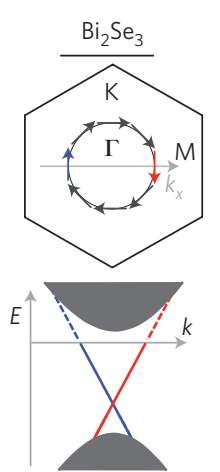

e

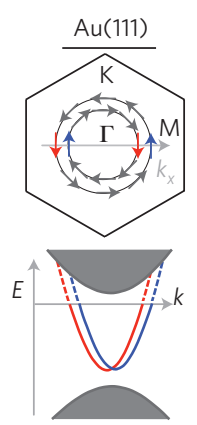

b

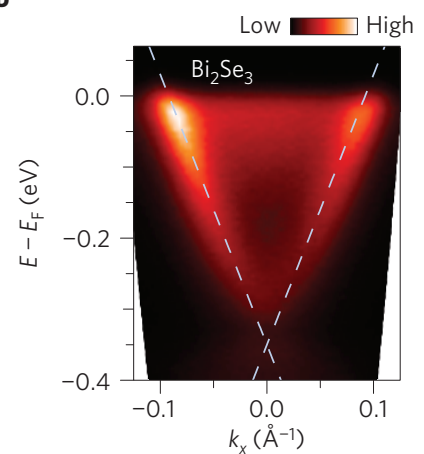

f

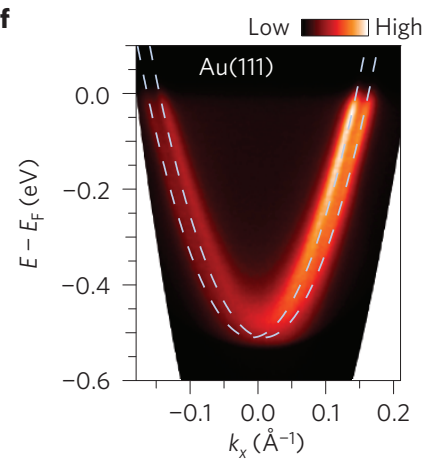

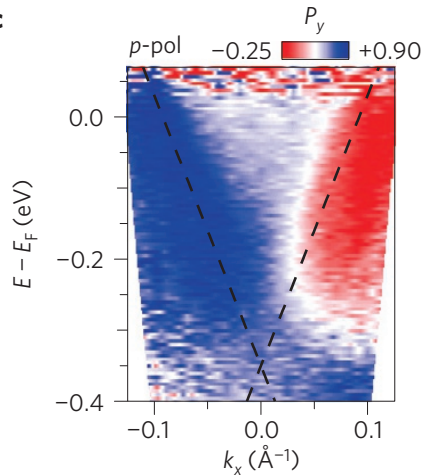

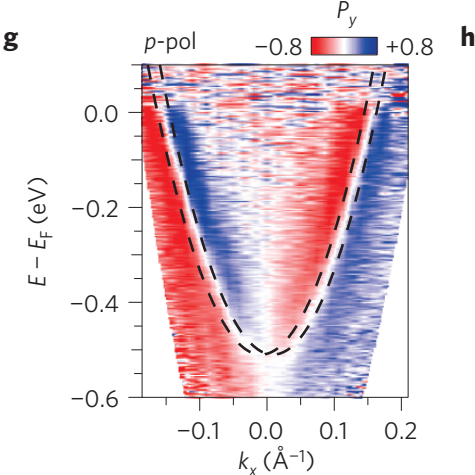

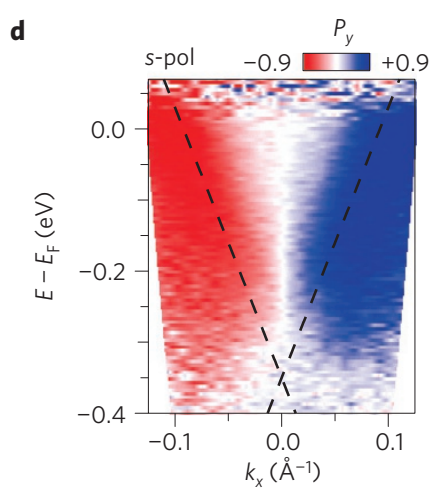

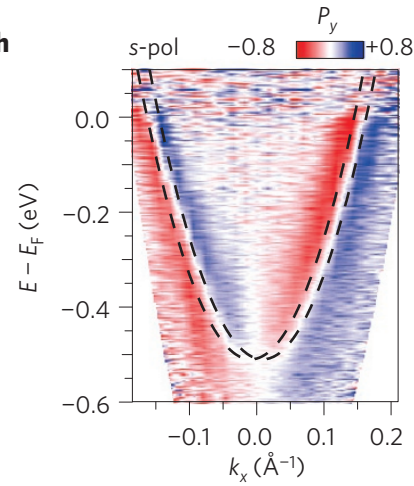

Figure 2 | Photoelectron spin flipping mapped through momentum space. a, Schematic of surface-state helical Dirac fermions in Bi $2 \mathrm{Se}_{3}$, including the Fermi surface (above) and the energy dispersion along $k_{x}$. b. Spin-integrated ARPES intensity map of $\mathrm{Bi}_{2} \mathrm{Se} \mathrm{z}_{3}$, taken with laser, $s$-polarized, $h \nu=5.99$ eV. Dashed lines are linear guides to the eye illustrating the Dirac cone dispersion of the surface state. $\mathbf{c}, \mathbf{d}$, Corresponding spin polarization $\left(P_{y}\right)$ maps taken with $p$ - and s-polarized light, respectively. Dashed guides to the eye are identical to $\mathbf{b}$. e-h, The same as in a-d, but for the Au(111) surface state. Dashed lines in $\mathbf{f - h}$ are parabolic guides to the eye following the free-electron-like dispersions.

spin-down (red), whereas those along the parabola shifted right are spin-up (blue), as expected ${ }^{24}$.

In contrast, when the light is $s$-polarized, the photoelectron spin polarization $\left(P_{y}\right)$ for $\mathrm{Bi}_{2} \mathrm{Se}_{3}$ is fully reversed (compare Fig. $2 \mathrm{c}$ and d), opposite to the expected surface-state electron spin texture. This is not the case for $\mathrm{Au}(111)$, where the spin polarization of photoelectrons is far less dependent on the light polarization (compare Fig. $2 \mathrm{~g}$ and $\mathrm{h}$ ), showing that the effects seen in $\mathrm{Bi}_{2} \mathrm{Se}_{3}$ are not generic or trivial experimental artefacts. This is true even for momentum points along both $k_{x}$ and $k_{y}$, measuring spin polarization along the $y$ and $z$ axes, and with both linear and circular light (see Supplementary Information).

The observations of strong dependence of the photoelectron spin polarization on photon polarization in $\mathrm{Bi}_{2} \mathrm{Se}_{3}$ demanded further investigation. Figure $3 \mathrm{a}, \mathrm{b}$ shows photoelectron spin polarization $\left(P_{y}\right)$ curves taken with $\pm s p$-polarized light, corresponding to the photon polarization vector being rotated to $\alpha_{0}= \pm 45^{\circ}$ (Fig. 1e), halfway between $p$ - and $s$-polarizations. Three $P_{y}$ curves are shown for each, corresponding to the three momentum locations along the $k_{y}$ axis shown in the inset diagrams. The theoretical surface-state electron spin texture predicts $P_{y}=0$ at momenta along the $k_{y}$ axis (that is, $k_{x}=0$ ) as the helical surface electrons are spin-polarized perpendicular to their momentum. As above, an overall k-independent positive shift in $P_{y}$ in the measurement is due to the particular experimental geometries in these cases (see Supplementary Information). The additional strong $k_{y}$ dependence in the data reveals the presence of a large radial component of the polarization, oriented as shown by the red and blue arrows in the diagrams, which was absent in previous measurements with $p$-polarized light ${ }^{12}$. Such a radial component of the photoelectron spin polarization differs from the expected surface-state electron spin texture that is primarily tangential at every point around the Fermi surface contour. It is also clear that the measured radial components reverse between $+s p$ - and $-s p$-polarized light geometries, again demonstrating control of the photoelectron spin polarization through the photon polarization.

This control extends to the out-of-plane dimension through the use of circularly polarized light as shown in Fig. 3c,d. Specifically, Fig. $3 \mathrm{c}$ shows a full map of photoelectron polarization, similar to Fig. 2c,d, but now measuring the out-of-plane spin component, $P_{z}$, and taken with right-hand circularly polarized light. Throughout the map, photoelectrons are primarily polarized with spins directed into the surface, reaching values of $P_{z}=-0.8$. Figure $3 \mathrm{~d}$ is a corresponding $P_{z}$ map taken with left-hand circularly polarized light, showing a full reversal with photoelectrons dominantly polarized with spin directed out of the surface, reaching values of $P_{z}=+0.8$.

The results shown in Figs 1-3 reveal the ability to fully manipulate the spin polarization of photoelectrons from a topological insulator through control of the light polarization, to an extent not previously observed in any system. They also illustrate non-equivalence of photoelectron and surface-state spins in a topological insulator, contrary to the usual assumption in spinresolved photoemission work. Indeed, the results in Figs 1 and 2 illustrate an interesting case of photoemission being dominated by a spin-flip process, an effect not experimentally observed before (see Supplementary Information).

The primary aspects of our results are well described by considering the case of light incident normal to the $\mathrm{Bi}_{2} \mathrm{Se}_{3}$ surface, wherein the interaction Hamiltonian of the surface-state electron and photon can be reduced to ${ }^{22}$

$$
H_{\text {int }} \propto(\sigma \times \hat{z}) \cdot \hat{\epsilon}
$$




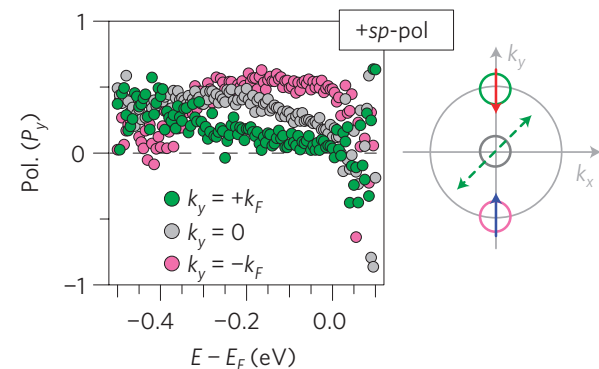

b

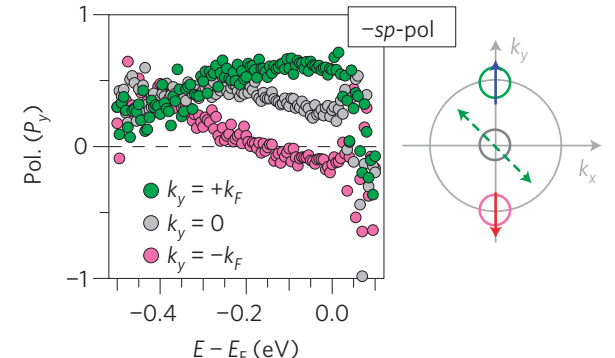

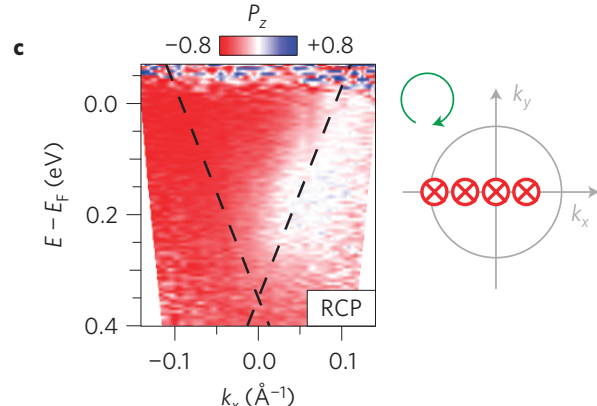

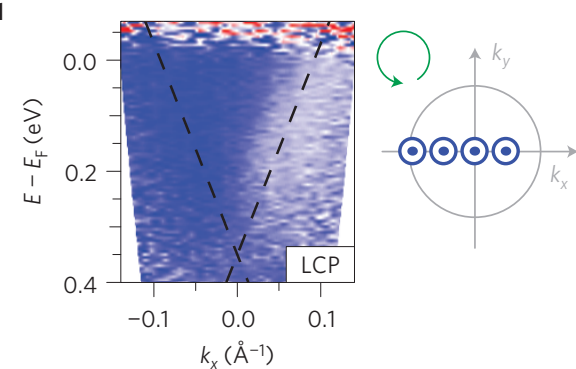

Figure 3 | $\mathrm{Bi}_{2} \mathrm{Se}_{3}$ photoelectron spin polarizations with \pm sp-polarized and circularly polarized light. a, Photoelectron $P_{y}$ curves at three values of $k_{y}$ along the $k_{y}$ axis, marked by small, colour-coded circles in insets, for $+s p$-polarized light, whose $\hat{\epsilon}$ projection in the surface plane is shown by the green arrow in the insets. $\mathbf{b}$. The same as in $\mathbf{a}$, but for $-s p$-polarized light. c, Photoelectron $P_{z}$ maps as a function of binding energy and momentum along the $k_{x}$ axis, with right-hand circularly polarized (RCP) light. The dashed lines are guides to the eye, marking the topological surface-state dispersion. $\mathbf{d}$, The same as in $\mathbf{c}$, but with left-hand circularly polarized (LCP) light.

where $\sigma$ is the Pauli spin matrix, $\hat{z}$ is the unit surface normal vector and $\hat{\epsilon}$ is the photon polarization vector. The presence of the Pauli spin operator readily shows that this interaction is capable of spin-flip transitions, counter to the usual assumption for such photoemission experiments. Calculations of the corresponding spin-dependent transition probabilities result in strong differences between predicted photoelectron spin polarization textures and the helical spin texture of the initial topological surface $s^{2} \mathrm{tat}^{22}$, as summarized in Fig. 4 for various photon polarizations, and in overall agreement with our measurements.

More specifically, the spin orientation of an electron in the helical surface state (Fig. 4a) can be expressed as

$$
\theta_{\mathbf{s}}=-\pi / 2+\theta_{\mathbf{k}}
$$

where $\theta_{\mathbf{s}}$ is the angle between the $+x$ direction and the spin direction at momentum $\mathbf{k}$, and $\theta_{\mathbf{k}}$ is the angle between the $+x$ direction and $\mathbf{k}$. In the case of linearly polarized light, with $\hat{\epsilon}$ parallel to the sample surface, the corresponding photoelectrons become spin polarized along directions given by ${ }^{22}$

$$
\theta_{\mathrm{s}}^{\prime}=-\pi / 2+\left(2 \alpha-\theta_{\mathbf{k}}\right)
$$

where $\alpha$ is the angle in the surface plane between the $+x$ direction and $\hat{\epsilon}$ (Fig. $4 \mathrm{~b}-\mathrm{e}$ ). Thus, there is a difference between the initial spin state and photoelectron spin polarizations at all momenta except for where $\theta_{\mathbf{k}}=\alpha$. Measurements within this typical geometry, such as Figs $1 \mathrm{c}$ and $2 \mathrm{c}$, have $\theta_{\mathrm{s}}^{\prime}=\theta_{\mathrm{s}}$. This is probably why previous spin-resolved ARPES works did not find results counter to the expected surface-state spin texture.

Equation (2) well describes the observed reversal of the photoelectron $P_{y}$ from $p$-polarized $(\alpha=0)$ to $s$-polarized $(\alpha=\pi / 2)$ light (Figs 1c,d and $2 \mathrm{c}, \mathrm{d}$ ), and the general $\cos (2 \alpha)$ dependence of the photoelectron $P_{y}$ measured at $\theta_{\mathbf{k}}=\pi$ (Fig. 1f). It also accounts for the large $P_{y}$ measurements of photoelectrons at $\theta_{\mathbf{k}}=\pi / 2$ and $3 \pi / 2$ measured with $\pm s p$-polarized light with $\alpha \sim \pm \pi / 4$ (compare Fig. 3a,b with Fig. 4d,e).
The above discussion leading to equation (2) assumes normally incident light, ignoring any possible component of the photon polarization along the out-of-surface-plane direction. Any such component introduces a spin-conserving term to the interaction Hamiltonian in equation (1) (ref. 22), which generally reduces the magnitude of the observed photon polarization dependence of the photoelectron spin polarization. In some cases, interference of the spin-flipping and -conserving terms results in photoelectron spin polarizations significantly different from when only one of them is present, consistent with results (see Supplementary Information).

Furthermore, these calculations predict normal-incidence circularly polarized light to result in the out-of-plane-directed photoelectron spin polarization textures shown in Fig. $4 \mathrm{f}, \mathrm{g}^{22}$. This is in general agreement with the present data shown in Fig. 3c,d. In both maps, however, a reduction in $P_{z}$ follows the surface-state dispersion at positive $k_{x}$ values. This is probably due in part to the off-normal photon incidence in the experiment (Fig. 1e) and that positive $k_{x}$ values are measured by having the sample normal rotated farther away from the incident light direction (see Supplementary Information). The results in Fig. $3 \mathrm{c}, \mathrm{d}$ are also in line with a recent theoretical study of photoemission from a related material, $\mathrm{Bi}_{2} \mathrm{Te}_{3}$ (ref. 25).

The observed photon-polarization-dependent photoelectron spin-flipping and spin-texture control in $\mathrm{Bi}_{2} \mathrm{Se}_{3}$ thus stems in part from strong spin-orbit coupling in the material. The observed absence of these effects for the $\mathrm{Au}(111)$ surface states (Fig. 2 and Supplementary Information), despite predictions of similar photoemission effects ${ }^{26}$, may be due to weaker spin-orbit coupling and the resulting dominance of a further spin-conserving term in the interaction Hamiltonian that is related to the inversion symmetry breaking at the surface ${ }^{27}$ (see Supplementary Information).

We hope our findings stimulate further studies of these effects and investigations of the material parameters required to observe them. Experimental insights may be gained through systematic studies of material systems with variable spin-orbit coupling such as $\operatorname{BiTl}\left(\mathrm{S}_{1}-\delta \mathrm{Se}_{\delta}\right)_{2}$ (ref. 11) and the adsorbate-induced Rashba states on $\mathrm{Bi}_{2} \mathrm{Se}_{3}$ (ref. 28), or other systems with exceptionally strong 


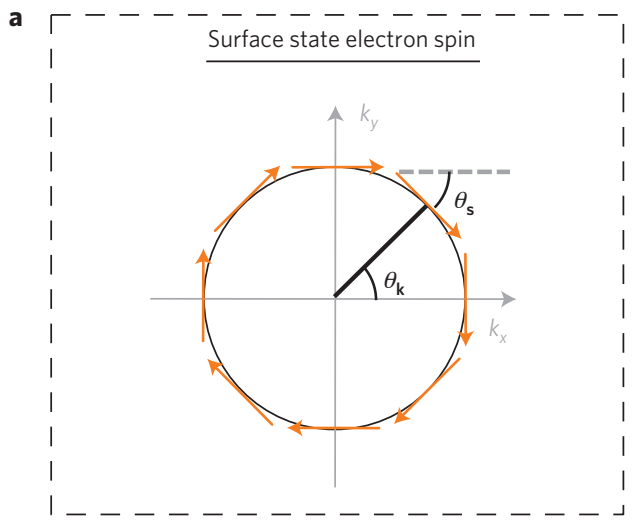

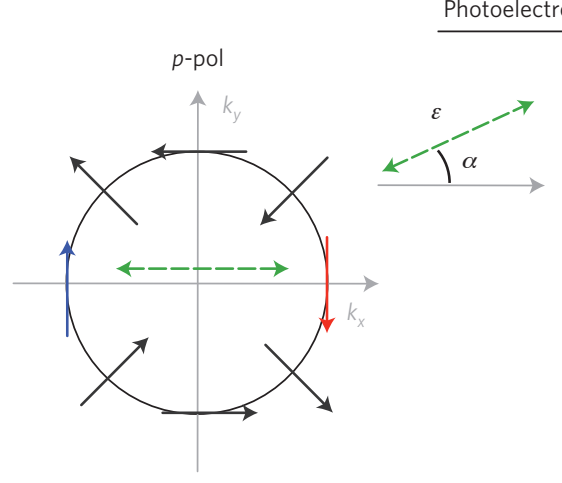

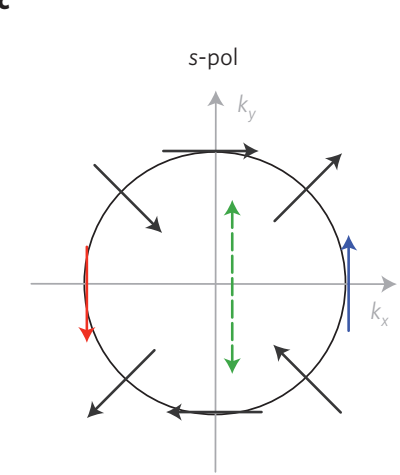

d

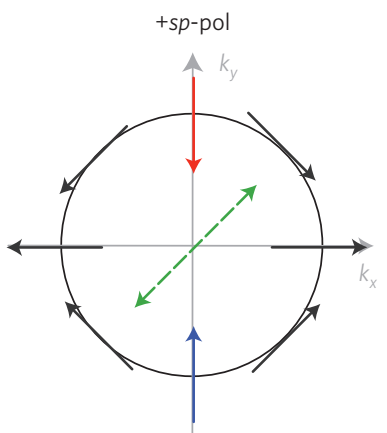

e

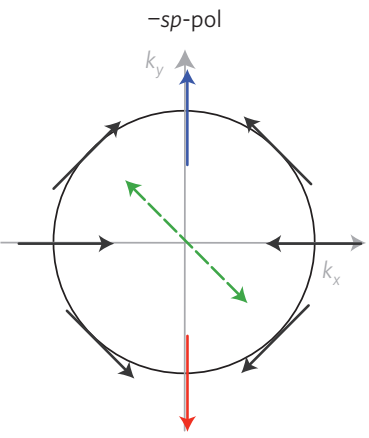

$\mathbf{f}$

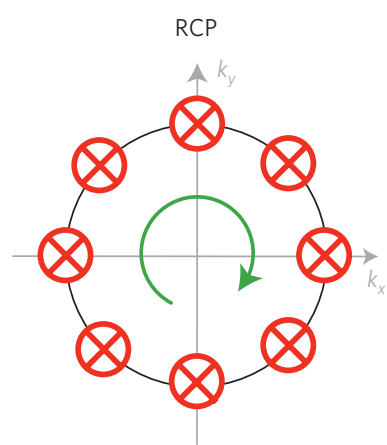

g

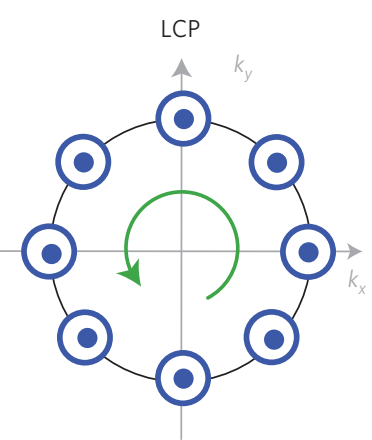

Figure 4 | Calculated photoelectron spin textures from a topological insulator for various photon polarizations. a, Spin texture of topological spin-helical Dirac electrons. Arrows depict spin of surface-state electrons, before photoemission. $\mathbf{b}-\mathbf{e}$, Calculated spin texture of photoelectrons from the same topological states, for various photon polarizations ${ }^{22}$. Arrows depict the photoelectron spin polarization directions, using linearly polarized light. The dashed green arrows mark the polarization vector, $\hat{\epsilon}$, projected onto the sample surface. These correspond to $p$-polarized (b), s-polarized (c) and \pm sp-polarized light (d,e) in the present experiment. The blue and red arrows correspond to the momentum positions and polarization directions consistent with the experimental data. b-e give equivalent information; their rotated orientations are meant to aid comparison with the experimental geometries. $\mathbf{f}, \mathbf{g}$, The same as in $\mathbf{b}-\mathbf{e}$, but with normally incident circularly polarized photons. Here, red crosses and blue dots depict photoelectron polarization into and out of the page along the $z$ axis. RCP, right-hand circularly polarized; LCP, left-hand circularly polarized.

spin-orbit coupling such as BiTeI (ref. 29). Further insights may be possible with other strongly spin-orbit coupled systems, such as $\mathrm{Bi}_{1-x} \mathrm{Sb}_{x}$ (ref. 7), in which the surface states extend to higher momenta where $k \cdot p$ theory is no longer valid and higher-order effects may come into play.

Independent of interpretation, our results demonstrate complete manipulation and control of photoelectron spin polarization from $\mathrm{Bi}_{2} \mathrm{Se}_{3}$. This could be used in a variety of applications ranging from spintronics to photocathode sources of polarized electron beams. Although a detailed study for evaluating and maximizing the photoconversion quantum efficiency for the latter application is necessary, the present results suggest that in comparison with the widely used GaAs photocathode ${ }^{30}, \mathrm{Bi}_{2} \mathrm{Se}_{3}$ could provide larger polarizations and enhanced functionality with complete control of spin orientation in three dimensions.

\section{Methods}

Experiments were performed on $\mathrm{Bi}_{2} \mathrm{Se}_{3}$ single crystals grown by directional slow solidification in an inclined ampoule and cleaved in situ along the (111) plane in a vacuum of $5 \times 10^{-11}$ torr. The $\mathrm{Au}(111)$ surface was prepared by in situ evaporation on a clean W(110) substrate according to standard methods. High-resolution spin-integrated ARPES data (Fig. 1b,c) were taken at beamline 4.0.3 at the Advanced Light Source with $35 \mathrm{eV}$ linearly $p$-polarized photons, at a sample temperature of $<20 \mathrm{~K}$. The energy and momentum resolutions were $\sim 13 \mathrm{meV}$ and $0.005 \AA^{-1}$, respectively. Spin-resolved ARPES data were taken with $5.99 \mathrm{eV}$ laser light and a high-efficiency spin-resolved spectrometer using time-of-flight and low-energy exchange-scattering techniques ${ }^{13}$. These data were taken at a sample temperature of $\sim 80 \mathrm{~K}$, with instrumental energy and momentum resolutions of $\sim 15 \mathrm{meV}$ and $0.02 \AA^{-1}$, respectively. The spectrometer acquires data as a function of binding energy in parallel, allowing high-resolution full EDCs to be acquired in 2-3 min, as opposed to several hours with conventional spin-resolved ARPES systems, thus precluding any surface-ageing effects (for example, vacuum or laser exposure) during acquisition and enabling the wide coverage of experimental parameter space in the experiment. Full two-dimensional energy-momentum polarization maps (Figs 2c,d,g,h and 3b,c) are made up of 20-30 individual EDCs. Each pair of maps (for example, Fig. 2c,d) is taken simultaneously, alternating the photon polarization after each EDC, such that photon polarization dependence in a pair of maps cannot be due to surface ageing. The momentum, or $\mathbf{k}$-vector probed in an EDC is scanned by rotating the crystal about the $y$ or $x$ axes, while the photon beam, photoelectron collection angle and spin analysis axis are all held fixed, as shown in Fig. 1e.

Received 26 September 2012; accepted 21 January 2013; published online 10 March 2013; corrected online 13 March 2013

\section{References}

1. Fu, L., Kane, C. L. \& Mele, E. J. Topological insulators in three dimensions. Phys. Rev. Lett. 98, 106803 (2007).

2. Moore, J. E. \& Balents, L. Topological invariants of time-reversal-invariant band structures. Phys. Rev. B 75, 121306 (2007).

3. Roy, R. Topological phases and the quantum spin Hall effect in three dimensions. Phys. Rev. B 79, 195322 (2009).

4. Qi, X-L. \& Zhang, S-C. The quantum spin Hall effect and topological insulators. Phys. Today 63, 33-38 (2010).

5. Moore, J. E. The birth of topological insulators. Nature 464, 194-198 (2010).

6. Hsieh, D. et al. A tunable topological insulator in the spin helical Dirac transport regime. Nature 460, 1101-1105 (2009).

7. Hsieh, D. et al. Observation of unconventional quantum spin textures in topological insulators. Science 323, 919-922 (2009).

8. Nishide, A. et al. Direct mapping of the spin-filtered surface bands of a three-dimensional quantum spin Hall insulator. Phys. Rev. B 81, 041309 (2010). 
9. Pan, Z-H. et al. Electronic structure of the topological insulator $\mathrm{Bi}_{2} \mathrm{Se}_{3}$ using angle-resolved photoemission spectroscopy: Evidence for a nearly full surface spin polarization. Phys. Rev. Lett. 106, 257004 (2011).

10. Souma, S. et al. Direct measurement of the out-of-plane spin texture in the Dirac-cone surface state of a topological insulator. Phys. Rev. Lett. 106, 216803 (2011)

11. Xu, S-Y. et al. Topological phase transition and texture inversion in a tunable topological insulator. Science 332, 560-564 (2011).

12. Jozwiak, C. et al. Widespread spin polarization effects in photoemission from topological insulators. Phys. Rev. B 84, 165113 (2011).

13. Jozwiak, C. et al. A high-efficiency spin-resolved photoemission spectrometer combining time-of-flight spectroscopy with exchange-scattering polarimetry. Rev. Sci. Instrum. 81, 053904 (2010).

14. Qi, X-L., Hughes, T. L. \& Zhang, S-C. Topological field theory of time-reversal invariant insulators. Phys. Rev. B 78, 195424 (2008).

15. Fu, L. \& Kane, C. L. Superconducting proximity effect and Majorana fermions at the surface of a topological insulator. Phys. Rev. Lett. 100, 096407 (2008).

16. Nayak, C., Simon, S. H., Stern, A., Freedman, M. \& Das Sarma, S. Non-Abelian anyons and topological quantum computation. Rev. Mod. Phys. 80, 1083-1159 (2008)

17. Roushan, P. et al. Topological surface states protected from backscattering by chiral spin texture. Nature 460, 1106-1109 (2009).

18. Peng, H. et al. Aharonov-Bohm interference in topological insulator nanoribbons. Nature Mater. 9, 225-229 (2010).

19. Hsieh, D. et al. Nonlinear optical probe of tunable surface electrons on a topological insulator. Phys. Rev. Lett. 106, 057401 (2011).

20. McIver, J. W., Hsieh, D., Steinberg, H., Jarillo-Herrero, P. \& Gedik, N. Control over topological insulator photocurrents with light polarization. Nature 7, 96-100 (2012).

21. Zhang, H. et al. Topological insulators in $\mathrm{Bi}_{2} \mathrm{Se}_{3}, \mathrm{Bi}_{2} \mathrm{Te}_{3}$ and $\mathrm{Sb}_{2} \mathrm{Te}_{3}$ with a single Dirac cone on the surface. Nature Phys. 5, 438-442 (2009).

22. Park, C-H. \& Louie, S. G. Spin polarization of photoelectrons from topological insulators. Phys. Rev. Lett. 109, 097601 (2012).

23. Xia, Y. et al. Observation of a large-gap topological-insulator class with a single Dirac cone on the surface. Nature Phys. 5, 398-402 (2009).

24. Hoesch, M. et al. Spin structure of the Shockley surface state on Au(111). Phys. Rev. B 69, 241401 (2004).

25. Mirhosseini, H. \& Henk, J. Spin texture and circular dichroism in photoelectron spectroscopy from the topological insulator $\mathrm{Bi}_{2} \mathrm{Te}_{3}$ : First-principles photoemission calculations. Phys. Rev. Lett. 109, 036803 (2012).
26. Henk, J., Ernst, A. \& Bruno, P. Spin polarization of the L-gap surface states on Au(11). Phys. Rev. B 68, 165416 (2003).

27. Kim, B. et al. Spin and orbital angular momentum structure of $\mathrm{Cu}(111)$ and $\mathrm{Au}(111)$ surface states. Phys. Rev. B 85, 195402 (2012).

28. King, P. D. C. et al. Large tunable Rashba spin splitting of a two-dimensional electron gas in $\mathrm{Bi}_{2} \mathrm{Se}_{3}$. Phys. Rev. Lett. 107, 096802 (2011).

29. Ishizaka, K. et al. Giant Rashba-type spin splitting in bulk BiTeI. Nature Mater. 10, 521-526 (2011).

30. Pierce, D. T. et al. The GaAs spin polarized electron source. Rev. Sci. Instrum. 51, 478-499 (1980)

\section{Acknowledgements}

We thank G. Lebedev and W. Wan for work with the electron optics, W. Zhang, D. A. Siegel, C. L. Smallwood and T. Miller for useful discussions, H. Wang and R. A. Kaindl for advice with optics, and A. Bostwick for help with software development. This work was supported by the Director, Office of Science, Office of Basic Energy Sciences, Division of Materials Sciences and Engineering, of the US Department of Energy under Contract No. DE-AC02-05CH11231 (Lawrence Berkeley National Laboratory). Higher-photon-energy photoemission work was performed at the Advanced Light Source, Lawrence Berkeley National Laboratory, which is supported by the Director Office of Science, Office of Basic Energy Sciences, of the US Department of Energy under Contract No. DE-AC02-05CH11231.

\section{Author contributions}

C.J. developed the experimental system. C.J., C-H.P. and C.H. devised the experiment. C.J. and K.G. carried out the experiment. C.J. analysed the experimental data. Calculations were performed by C-H.P., S.G.L. and D-H. L. Samples were prepared by C.R.R. and R.J.B. Synchrotron data were acquired by J.D.D., C.J. and K.G. Z.H. and A.L. were responsible for experiment planning and infrastructure. All authors contributed to the interpretation and writing of the manuscript.

\section{Additional information}

Supplementary information is available in the online version of the paper. Reprints and permissions information is available online at www.nature.com/reprints. Correspondence and requests for materials should be addressed to A.L.

\section{Competing financial interests}

The authors declare no competing financial interests. 